UR-1361

ER40685-811

\title{
ON THE WARD IDENTITIES AT FINITE TEMPERATURE
}

\author{
Ashok Das \\ and \\ Marcelo Hott ${ }^{\dagger}$ \\ Department of Physics and Astronomy \\ University of Rochester \\ Rochester, NY 14627
}

\begin{abstract}
$\underline{\text { Abstract }}$
We show both in $1+1$ and $3+1$ dimensions that, contrary to the recent suggestions, the contribution of the fermion loop to the polarization tensor is manifestly transverse at finite temperature. Some subtleties associated with the Ward identities at finite temperature are also pointed out.
\end{abstract}

†On leave of absence from UNESP - Campus de Guaratinguetá, P.O. Box 205, CEP : 12.500, Guaratinguetá, S.P., Brazil 


\section{Introduction:}

Studies in finite temperture field theory have been of much interest in recent years from various points of view [1-3]. There are three distinct formalisms for carrying out calculations at finite temperature, namely, the imaginary time formalism [4], thermofield dynamics [5] and the closed time path formalism [6]. While the nature of a finite temperature calculation, in each of these formalisms, is completely parallel to the zero temperature calculation, some of the resulting properties at finite temperature are quite distinct. For example, the analytic behavior of Feynman amplitudes at finite temperature is noticeably different from those at zero temperature [7-9], the infrared behavior at finite temperature is markedly more singular [10] and so on. As more and more studies are carried out, we will no doubt have a better understanding of the structure of quantum field theory at finite temperature.

It is commonly believed that gauge invariance is not affected by temperature. The recent suggestion that Ward identities may be violated at finite temperature [11] is, therefore, quite surprising. More specifically, it was argued that the contribution of the fermion loop, at finite temperature, to the polarization tensor is not transverse with $\epsilon$-regularization [8]. We note that the physical component of the fermion propagator at finite temperature has the form

$$
\begin{aligned}
S(p) & =S^{(0)}(p)+S^{(\beta)}(p) \\
& =i(\not p+m)\left(\frac{1}{p^{2}-m^{2}+i \epsilon}+2 i \pi n_{F}(p) \delta\left(p^{2}-m^{2}\right)\right)
\end{aligned}
$$

where

$$
n_{F}(p)=\frac{1}{e^{\beta\left|p_{0}\right|}+1}
$$

represents the fermion distribution function. (One can write this more covariantly by introducing the velocity of the heat bath, but we will ignore it in this initial discussion.) The temperature dependent contribution of the fermion loop 
to the polarization tensor is, then, given by ( $D$ is the number of space-time dimensions)

$$
\begin{aligned}
&-i \Pi_{\mu \nu}^{(\beta)}(p)= e^{2} \int \frac{d^{D} k}{(2 \pi)^{D}} \operatorname{Tr}\left[\gamma_{\mu} S^{(0)}(k+p) \gamma_{\nu} S^{(\beta)}(k)+\gamma_{\mu} S^{(\beta)}(k+p) \gamma_{\nu} S^{(0)}(k)\right. \\
&\left.+\gamma_{\mu} S^{(\beta)}(k+p) \gamma_{\nu} S^{(\beta)}(k)\right] \\
&=-D e^{2} \int \frac{d^{D} k}{(2 \pi)^{D}}\left[(k+p)_{\mu} k_{\nu}+(k+p)_{\nu} k_{\mu}-\eta_{\mu \nu}\left(k \cdot(k+p)-m^{2}\right)\right] \\
& \times\left[\frac{2 i \pi n_{F}(k)}{(k+p)^{2}-m^{2}+i \epsilon} \delta\left(k^{2}-m^{2}\right)+\frac{2 i \pi n_{F}(k+p)}{k^{2}-m^{2}+i \epsilon} \delta\left((k+p)^{2}-m^{2}\right)\right. \\
&\left.-4 \pi^{2} n_{F}(k) n_{F}(k+p) \delta\left(k^{2}-m^{2}\right) \delta\left((k+p)^{2}-m^{2}\right)\right]
\end{aligned}
$$

The three terms in $p^{\mu} \Pi_{\mu \nu}^{(\beta)}(p)$ can now be easily seen to vanish individually due to the $\delta$-function constraints and the symmetry of the respective integrands. It is, therefore, even more surprising that an explicit evaluation of the polarization tensor will be nontransverse considering that temperature dependent contributions are finite.

In this paper, we report on a systematic study of the transverse nature of the fermion loop contribution to the polarization tensor at finite temperature. In sec. II, we recapitulate various known facts about distinct tensor structures at finite temperature. In sec. III, we show that in $1+1$ dimensions, the fermion bubble has a transverse tensor structure which factors out of the integral. In sec. IV, we show that the integrand for the fermion bubble is manifestly transverse even in $3+1$ dimensions. In sec. V, we point out some subtleties associated with the Ward identities at finite temperature and present our conclusions in sec. VI.

\section{Tensor Structures at Finite $T$ :}

In this brief section, we collect all the known facts [12] about various tensor structures at finite temperature for completeness as well as for later use. We note that a covariant 
description, at finite temperature, involves the velocity of the heat bath, $u^{\mu}$, in addition to the available four momenta. Any vector can, of course, be decomposed into components parallel and perpendicular to $u^{\mu}$ as $\left(u^{2}=u^{\mu} u_{\mu}=1\right)$

$$
\begin{aligned}
& A_{\|}^{\mu}=(A \cdot u) u^{\mu} \\
& A_{\perp}^{\mu}=\widetilde{A}^{\mu}=A^{\mu}-(A \cdot u) u^{\mu}
\end{aligned}
$$

In particular, we note that for a given momentum $p^{\mu}$, if we define

$$
\omega=(p \cdot u)
$$

Then, the component of $p^{\mu}$ orthogonal to $u^{\mu}$ will be given by

$$
\widetilde{p}^{\mu}=p^{\mu}-\omega u^{\mu}
$$

It follows from this that

$$
\widetilde{p}^{2}=\widetilde{p}^{\mu} \widetilde{p}_{\mu}=p^{2}-\omega^{2}
$$

From Eq. (4), it is clear that the projection operator onto the space of vectors transverse to $u^{\mu}$ is given by

$$
\widetilde{\eta}^{\mu \nu}=\eta^{\mu \nu}-u^{\mu} u^{\nu}
$$

Here $\eta^{\mu \nu}$ is the metric and in our convention has the signature $(+,-,-,-)$.

One can, of course, decompose any vector with respect to a given momentum $p^{\mu}$ as well. Thus, the component of a vector $A^{\mu}$, transverse to $p^{\mu}$ is given by

$$
\bar{A}^{\mu}=A^{\mu}-\frac{1}{p^{2}}(p \cdot A) p^{\mu}
$$

In particular, this gives

$$
\bar{u}^{\mu}=u^{\mu}-\frac{\omega}{p^{2}} p^{\mu}
$$

which is orthogonal to $p^{\mu}$. Note, from Eq. (9), that the projection operator onto the space of vectors orthogonal to $p^{\mu}$ is given by

$$
\bar{\eta}^{\mu \nu}=\eta^{\mu \nu}-\frac{p^{\mu} p^{\nu}}{p^{2}}
$$


Given these, it is easy to check that at finite temperature, we can construct only three second rank, symmetric tensors from $p^{\mu}$ and $u^{\mu}$ which are orthogonal to $p^{\mu}$, namely,

$$
\begin{aligned}
& A^{\mu \nu}=\eta^{\mu \nu}-\frac{p^{\mu} p^{\nu}}{p^{2}} \\
& B^{\mu \nu}=\widetilde{\eta}^{\mu \nu}-\frac{\widetilde{p}^{\mu} \widetilde{p}^{\nu}}{\widetilde{p}^{2}} \\
& C^{\mu \nu}=\frac{p^{2}}{\widetilde{p}^{2}} \bar{u}^{\mu} \bar{u}^{\nu}
\end{aligned}
$$

Each of these structures is normalized so that they act like projection operators. In fact, they satisfy

$$
\begin{array}{lll}
A^{\mu \nu} A_{\nu \lambda}=A_{\lambda}^{\mu} & B^{\mu \nu} B_{\nu \lambda}=B_{\lambda}^{\mu} & C^{\mu \nu} C_{\nu \lambda}=C_{\lambda}^{\mu} \\
A^{\mu \nu} B_{\nu \lambda}=B_{\lambda}^{\mu} & A^{\mu \nu} C_{\nu \lambda}=C_{\lambda}^{\mu} & B^{\mu \nu} C_{\nu \lambda}=0
\end{array}
$$

It is also easy to check that the three structures are not independent. In fact,

$$
A^{\mu \nu}=B^{\mu \nu}+C^{\mu \nu}
$$

and since $B^{\mu \nu}$ and $C^{\mu \nu}$ are orthogonal to each other, it is more useful to treat them as independent. Given this, then, one can write down the most general transverse polarization tensor at finite temperature to have the form

$$
\begin{aligned}
\Pi_{\mu \nu}^{(\beta)}(p) & =B_{\mu \nu} \Pi_{T}^{(\beta)}(p)+C_{\mu \nu} \Pi_{L}^{(\beta)}(p) \\
& =\left(\widetilde{\eta}_{\mu \nu}-\frac{\widetilde{p}_{\mu} \widetilde{p}_{\nu}}{\widetilde{p}^{2}}\right) \Pi_{T}^{(\beta)}(p)+\frac{p^{2}}{\widetilde{p}^{2}} \bar{u}_{\mu} \bar{u}_{\nu} \Pi_{L}^{(\beta)}(p)
\end{aligned}
$$

At zero temperature, $\Pi_{T}(p)=\Pi_{L}(p)$ and with the identity (14), we see that the polarization tensor reduces to the usual structure. It is also easy to check from Eq. (15) that

$$
\begin{aligned}
\Pi_{L}^{(\beta)} & =\frac{p^{2}}{\widetilde{p}^{2}} u^{\mu} u^{\nu} \Pi_{\mu \nu}^{(\beta)} \\
(D-2) \Pi_{T}^{(\beta)} & =\eta^{\mu \nu} \Pi_{\mu \nu}-\Pi_{L}^{(\beta)}
\end{aligned}
$$

where $D$ is the number of space-time dimensions. We note that Eq. (16) does not yield any information on $\Pi_{T}^{(\beta)}$ when $D=2$. 


\section{Polariation Tensor in $1+1$ Dimensions:}

In this section, we study the contribution of the fermion bubble to the polarization tensor at finite temperature in $1+1$ dimensions. From Eq. (3), we note that

$$
\begin{aligned}
-i \Pi_{\mu \nu}^{(\beta)}(p)= & -\frac{i e^{2}}{\pi} \int d^{2} k\left[(k+p)_{\mu} k_{\nu}+(k+p)_{\nu} k_{\mu}-\eta_{\mu \nu}\left(k \cdot(k+p)-m^{2}\right)\right] \\
& \times\left[\frac{n_{F}(k)}{(k+p)^{2}-m^{2}+i \epsilon} \delta\left(k^{2}-m^{2}\right)+\frac{n_{F}(k+p)}{k^{2}-m^{2}+i \epsilon} \delta\left((k+p)^{2}-m^{2}\right)\right. \\
& \left.+2 i \pi n_{F}(k) n_{F}(k+p) \delta\left(k^{2}-m^{2}\right) \delta\left((k+p)^{2}-m^{2}\right)\right]
\end{aligned}
$$

where the covariant fermion distribution function is given by

$$
n_{F}(k)=\frac{1}{e^{\beta|u \cdot k|}+1}
$$

The simplicity of $1+1$ dimensions allows one to factor the tensor structure out of the integral. To see this, let us define

$$
\begin{aligned}
\Omega & =k \cdot u \quad \omega=p \cdot u \\
k^{\mu} & =\Omega u^{\mu}-\epsilon^{\mu \nu} u_{\nu} k^{\prime} \\
p^{\mu} & =\omega u^{\mu}-\epsilon^{\mu \nu} u_{\nu} p^{\prime}
\end{aligned}
$$

In our notation $\epsilon^{01}=1$ and $k^{\prime 2}=-\widetilde{k}^{2}$. Substituting these new variables into Eq. (17) (The Jacobian can be easily checked to be unity.) and with some straightforward algebraic manipulations we find that the $\delta$-function constraints as well as the symmetry properties of the integrals give

$$
-i \Pi_{\mu \nu}^{(\beta)}(p)=-e^{2} \bar{u}_{\mu} \bar{u}_{\nu}(2 I+\widetilde{I})
$$

where, in $1+1$ dimensions, we have

$$
\bar{u}_{\mu}=u_{\mu}-\frac{\omega}{p^{\prime}} \epsilon_{\mu \nu} u^{\nu}
$$

and

$$
\begin{gathered}
I=\frac{i p^{\prime}}{\omega} \int d \Omega d k^{\prime} \frac{k^{\prime}(\omega+\Omega)+\Omega\left(k^{\prime}+p^{\prime}\right)}{\omega(\omega+2 \Omega)-p^{\prime}\left(p^{\prime}+2 k^{\prime}\right)+i \epsilon} \frac{1}{e^{\beta|\Omega|}+1} \delta\left(\Omega^{2}-k^{2}-m^{2}\right) \\
\widetilde{I}=-\frac{2 p^{\prime}}{\omega} \int d \Omega d k^{\prime}\left(k^{\prime}(\omega+\Omega)+\Omega\left(k^{\prime}+p^{\prime}\right)\right) \frac{1}{e^{\beta|\Omega|}+1} \frac{1}{e^{\beta|\omega+\Omega|}+1} \delta\left(\Omega^{2}-k^{\prime 2}-m^{2}\right) \\
\times \delta\left((\Omega+\omega)^{2}-\left(k^{\prime}+p^{\prime}\right)^{2}-m^{2}\right)
\end{gathered}
$$


We note that the temperature dependent part of the polarization tensor is explicitly transverse even before evaluating the integrals and, therefore, any prescription ( $\epsilon$-regularization, modified Feynman parameterization etc.) for evaluating the integral cannot change this. Comparing with Eq. (15), we note that in this case,

$$
\Pi_{T}^{(\beta)}(p)=0
$$

If $m=0$, then one can evaluate the integrals in Eq. (22) in a straightforward manner and show that

$$
\begin{aligned}
& \operatorname{Im} I=0 \\
& \operatorname{Re}(2 I+\widetilde{I})=0
\end{aligned}
$$

so that for $m=0$

$$
\begin{aligned}
& \Pi_{L}^{(\beta)}=0 \\
& \Pi_{\mu \nu}^{(\beta)}=0
\end{aligned}
$$

This is indeed consistent with what one knows about the Schwinger model [13 -15] where the temperature dependent corrections to the photon mass and the chiral anomaly vanish.

When $m \neq 0$, however, $\Pi_{L}^{(\beta)}$ does not vanish and gives the only temperature dependent correction to $\Pi_{\mu \nu}^{(\beta)}$. In this case it is easy to see that $\operatorname{Re} \Pi_{L}^{(\beta)}(p)$ is nonanalytic at $p^{\mu}=0$ much like the scalar theories.

\section{Polarization Tensor in $3+1$ Dimensions:}

The structure of the polarization tensor in $3+1$ dimensions is still the same as in $1+1$ dimensions, namely,

$$
\begin{aligned}
-i \Pi_{\mu \nu}^{(\beta)}=-\frac{i e^{2}}{2 \pi^{3}} \int d^{4} k\left[(k+p)_{\mu} k_{\nu}+(k+p)_{\nu} k_{\mu}-\eta_{\mu \nu}\left(k \cdot(k+p)-m^{2}\right)\right] \\
\times\left[\frac{n_{F}(k)}{(k+p)^{2}-m^{2}+i \epsilon} \delta\left(k^{2}-m^{2}\right)+\frac{n_{F}(k+p)}{k^{2}-m^{2}+i \epsilon} \delta\left((k+p)^{2}-m^{2}\right)\right. \\
\left.\quad+2 i \pi n_{F}(k) n_{F}(k+p) \delta\left(k^{2}-m^{2}\right) \delta\left((k+p)^{2}-m^{2}\right)\right]
\end{aligned}
$$

However, in $3+1$ dimensions, it is not possible to factor the tensor structure out of the integral [16]. 
To proceed in this case, therefore, we decompose vectors into components parallel and perpendicular to $p^{\mu}$. Thus, let (This is different from $\Omega$ of the last section.)

$$
\begin{aligned}
\Omega & =p \cdot k \quad \omega=u \cdot p \\
k^{\mu} & =\Omega \frac{p^{\mu}}{p^{2}}+\bar{k}^{\mu} \\
u^{\mu} & =\frac{\omega}{p^{2}} p^{\mu}+\bar{u}^{\mu}
\end{aligned}
$$

It is clear that $\bar{k}^{\mu}$ is manifestly transverse to $p^{\mu}$. Substituting this back into Eq. (26) and using the $\delta$-function constraint as well as the symmetry properties of the integrals, we obtain

$$
\begin{aligned}
-i \Pi_{\mu \nu}^{(\beta)}(p)= & -\frac{i e^{2}}{\pi^{3}} \int d \Omega d^{4} \bar{k} \delta(p \cdot \bar{k})\left[\left(-\eta_{\mu \nu}+\frac{p_{\mu} p_{\nu}}{p^{2}}\right) \frac{\Omega}{2}+\bar{k}_{\mu} \bar{k}_{\nu}\right] \\
& \times\left[\frac{2}{p^{2}+2 \Omega+i \epsilon} \frac{1}{e^{\beta\left|\frac{\omega \Omega}{p^{2}}+u \cdot \bar{k}\right|}+1} \delta\left(\frac{\Omega^{2}}{p^{2}}+\bar{k}^{2}-m^{2}\right)\right. \\
& +2 i \pi \frac{1}{e^{\beta\left|\frac{\omega \Omega}{p^{2}}+u \cdot \bar{k}\right|}+1} \frac{1}{e^{\beta\left|\omega\left(1+\frac{\Omega}{p^{2}}\right)+u \cdot \bar{k}\right|}+1} \\
& \left.\times \delta\left(\frac{\Omega^{2}}{p^{2}}+\bar{k}^{2}-m^{2}\right) \delta\left(p^{2}+2 \Omega\right)\right]
\end{aligned}
$$

We note that the first tensor structure is nothing other than $A_{\mu \nu}=B_{\mu \nu}+C_{\mu \nu}$ and can be factored out of the integral. While the second structure cannot be factored out of the integral, we note that it is manifestly orthogonal to $p^{\mu}$. Thus, independent of the prescription used to evaluate this, it can only lead to a linear combination of $B_{\mu \nu}$ and $C_{\mu \nu}$. Thus, we see that the temperature dependent contribution of the fermion bubble to the polarization tensor continues to be manifestly transverse even in $3+1$ dimensions. Contrary to the $1+1$ dimensions, however, in the present case both $\Pi_{T}^{(\beta)}$ and $\Pi_{L}^{(\beta)}$ are a priori nonvanishing.

\section{Observations on Ward Identities:}

At zero temperature, the Ward identities of QED can be written in several equivalent ways. For example, if

$$
S^{(0)}(p)=\frac{i}{\not p-m+i \epsilon}
$$

denotes the tree level fermion propagator at zero temperature, then

$$
\frac{\partial S^{(0)}(p)}{\partial p^{\mu}}=-\frac{1}{e} S^{(0)}(p) \Gamma_{\mu}^{(0)} S^{(0)}(p)
$$


where

$$
\Gamma_{\mu}^{(0)}=-i e \gamma_{\mu}
$$

is the vertex at the tree level. The identity in Eq. (30) can be shown to hold order by order so that to all orders one can write

$$
\frac{\partial S(p)}{\partial p^{\mu}}=-\frac{1}{e} S(p) \Gamma_{\mu} S(p)
$$

where $S(p)$ and $\Gamma_{\mu}$ represent the complete fermion propagator and the vertex of the theory. This is one form of the Ward identity and, in fact, is the form in which it was originally derived [17]. The integrated form of Eq. (32) which can also be derived from the BRS invariance [18] of the theory is given by

$$
\left(p^{\prime}-p\right)^{\mu} S\left(p^{\prime}\right) \Gamma_{\mu} S(p)=e\left(S(p)-S\left(p^{\prime}\right)\right)
$$

At zero temperature both the forms of the Ward identity are equivalent.

At finite temperature, however, the propagators have a $2 \times 2$ matrix structure [5-6]. Thus, for example, the fermion propagator can be written as

$$
S(p)=\left(\begin{array}{cc}
S_{++}(p) & S_{+-}(p) \\
S_{-+}(p) & S_{--}(p)
\end{array}\right)
$$

where at tree level, the components of the matrix have the form

$$
\begin{aligned}
& S_{++}(p)=i(\not p+m)\left(\frac{1}{p^{2}-m^{2}+i \epsilon}+2 i \pi n_{F}(p) \delta\left(p^{2}-m^{2}\right)\right) \\
& S_{+-}(p)=i(\not p+m)\left(-2 i \pi \theta\left(-p^{0}\right)+2 i \pi n_{F}(p)\right) \delta\left(p^{2}-m^{2}\right) \\
& S_{-+}(p)=i(\not p+m)\left(-2 i \pi \theta\left(p^{0}\right)+2 i \pi n_{F}(p)\right) \delta\left(p^{2}-m^{2}\right) \\
& S_{--}(p)=i(\not p+m)\left(-\frac{1}{p^{2}-m^{2}+i \epsilon}+2 i \pi n_{F}(p) \delta\left(p^{2}-m^{2}\right)\right)
\end{aligned}
$$

The vertex at finite temperature also has a $2 \times 2$ matrix structure which at the tree level is given by

$$
\Gamma_{\mu}=-i e\left(\begin{array}{cc}
\gamma_{\mu} & 0 \\
0 & -\gamma_{\mu}
\end{array}\right)
$$

One can now check explicitly and show that the identity in Eq. (33) continues to hold even at finite temperature be it in terms of matrices. The identity in Eq. (30), however, 
modifies at finite temperature (as can be checked explicitly from the tree level functions defined in Eqs. (35) and (36)) to

$$
\frac{\partial S(p)}{\partial p^{\mu}}=-\frac{1}{e} S(p) \Gamma_{\mu} S(p)-2 \pi \frac{\partial n_{F}(p)}{\partial p^{\mu}}(\not p+m) \delta\left(p^{2}-m^{2}\right)\left(\begin{array}{ll}
1 & 1 \\
1 & 1
\end{array}\right)
$$

The additional term in Eq. (37), however, can be seen to vanish when multiplied by $(\not p-m)$ or $S^{-1}(p)$. Let us also note that the retarded and the advanced propagators defined as

$$
\begin{aligned}
& S_{R}(p)=S_{++}(p)-S_{+-}(p) \\
& S_{A}(p)=S_{++}(p)-S_{-+}(p)
\end{aligned}
$$

on the other hand, can be seen to satisfy

$$
\begin{aligned}
& \frac{\partial S_{R}(p)}{\partial p^{\mu}}=-S_{R}(p) \gamma_{\mu} S_{R}(p) \\
& \frac{\partial S_{A}(p)}{\partial p^{\mu}}=-S_{A}(p) \gamma_{\mu} S_{A}(p)
\end{aligned}
$$

without the extra term in Eq. (37). Thus, one should be careful at finite temperature in the use of the form of Ward identity.

\section{Conclusion:}

In this paper, we have systematically studied the transverse nature of the fermion bubble contribution to the polarization tensor at finite temperature. Contrary to the recent suggestions, we have shown that the polarization tensor is manifestly transverse. In $1+1$ dimensions the transverse tensor structure factors out of the integral and yields a nonvanishing contribution only to $\Pi_{L}^{(\beta)}$ when $m \neq 0$. In $3+1$ dimensions, the tensor structure does not factor out of the integral, but is manifestly transverse. We have also pointed out some subtleties associated with the Ward identities at finite temperature.

One of us (A.D.) would like to thank Prof. E. Witten and the Institute for Advanced Studies for hospitality where part of this work was done. This work was supported in part by the U.S. Department of Energy Grant No. DE-FG-02-91ER40685. M.H. would like to thank the Fundação de Amparo a Pesquisa do Estado de São Paulo for the financial support. 


\section{References:}

1. For recent reviews see N.P. Landsman and Ch. G. Van Weert, Phys. Rep. 145 (1987) 141; K.C. Chou, Z.B. Su, B.C. Hao and L. Yu, Phys. Rep. 118 (1985) 1; E. Calzetta, B.L. Hu and J.P. Paz, "Statistical and Kinetic Field Theory", to be published.

2. E. Braaten and R. Pisarski, Nucl. Phys. B337 (1990) 569; E. Braaten and R. Pisarski, Phys. Rev. D45 (1992) R1827; J. Frenkel and J.C. Taylor, Nucl. Phys. B334 (1990) 199; J.C. Taylor and S.M. Wong, Nucl. Phys. B346 (1990) 115.

3. A. Anselm, Phys. Lett. B217 (1989) 169; J.D. Bjorken, Int. J. Mod. Phys. A7 (1992) 4189; K.L. Kowalsky and C.C. Taylor, Case Western Reserve University preprint 92-6; K. Rajagopal and F. Wilczek, Nucl. Phys. B404 (1993) 577; P.F. Bedaque and A. Das, Mod. Phys. Lett. A8 (1993) 3151.

4. For an introduction see A.L. Fetter and J.D. Walecka, "Quantum Theory of Many Particle Systems", McGraw-Hill (1971); A.A. Abrikosov, L.P. Gorkov and I.E. Dzyaloshinski, "Methods of Quantum Field Theory in Statistical Physics", Dover (1963).

5. H. Umezawa, H. Matsumoto and M. Tachiki, "Thermo Field Dynamics and Condensed States", North Holland (1982).

6. J. Schwinger, J. Math. Phys. 2 (1961) 407; J. Schwinger, Lecture Notes of Brandeis Summer Institute in Theoretical Physics (1960); L.V. Keldysh, Sov. Phys. JETP 20 (1965) 1018.

7. For an excellent review of the problem and references see P.S. Gribosky and B.R. Holstein, Z. Phys. C47 (1990) 205.

8. P.F. Bedaque and A. Das, Phy. Rev. D45 (1992) 2906.

9. H.A. Weldon, Phys. Rev. D47 (1993) 594; P.F. Bedaque and A. Das, Phys. Rev. D47 (1993) 601.

10. For a review and references see H. Weldon, West Virginia University preprint hepph/9311245.

11. N.R. Pantoja, Universidad de los Andes preprint (1994).

12. We follow here mainly the discussion in H.A. Weldon, Phys. Rev. D26 (1982) 1394.

13. J. Schwinger, Phys. Rev. 128 (1962) 2425.

14. L. Dolan and R. Jackiw, Phys. Rev. D9 (1974) 3320. 
15. A. Das and A. Karev, Phys. Rev. D36 (1987) 623.

16. The polarization tensor has also been evaluated in ref. 12 and in O.K. Kalashinov and V.V. Klimov, Sov. J. Nucl. Phys. 31 (1980) 699.

17. J.C. Ward, Phys. Rev. 77 (1950) 293; ibid 78 (1950) 182; Y. Takahashi, Nuovo Cimento 6 (1957) 370.

18. C. Becchi, A. Rouet and R. Stora, Comm. Math. Phys. 52 (1975) 55. 